Research Article

\title{
Unmet need and limitations of current anticoagulation therapy for stroke prevention in atrial fibrillation patients: a questionnaire based study
}

\author{
Maulin Dhiren Mehta*
}

Department of Pharmacology, R. D. Gardi Medical College, Ujjain, Madhya Pradesh, India

Received: 19 May 2016 Accepted: 13 June 2016

*Correspondence to: Dr. Maulin Dhiren Mehta, Email:maulin_mehta@ rediffmail.com

Copyright: () the author(s), publisher and licensee Medip Academy. This is an openaccess article distributed under the terms of the Creative Commons Attribution NonCommercial License, which permits unrestricted noncommercial use, distribution, and reproduction in any medium, provided the original work is properly cited.

\begin{abstract}
Background: Atrial fibrillation (AF) is one of the commonest of cardiac arrhythmia. Non-valvular atrial fibrillation (NVAF) leads to increase in risks of stroke and death. The 'stroke prevention in atrial fibrillation' (SPAF) trial have shown that oral anticoagulation therapy (warfarin) is the most effective drug for SPAF. On the other side, warfarin is associated with the risk of serious bleeding. Complications associated with warfarin lead us to in urge of search for effective, safer and convenient newer oral anticoagulation therapy (NOAC). Methods: The study was observational, non-interventional and prospective questionnaire based, willingly participated by 20 cardiologists of Vadodara. It was done to assess the prevalence of NVAF, to find out unmet need and limitations of warfarin and future of newer oral anticoagulation drugs.

Results: According to cardiologists, prevalence of NVAF was $2.5-3 \%$ in vadodara, India. Prophylactic therapy for SPAF was given after evaluating CHA2DS2-VASc score and choice of therapy was warfarin for $60 \%$ of total cardiologists. Total $90 \%$ of the cardiologists mentioned that bleeding and continuous monitoring are the commonly encountered problems due to warfarin. According to all cardiologists, warfarin is unsafe due to its serious side effects and change is required. NOAC therapy without antidote can be accepted by all, if they are safe enough. Total $90 \%$ of cardiologists mentioned that NOAC drugs must have better safety with comparable efficacy to warfarin. Conclusions: Safety issues and continuous INR monitoring are the limitations of warfarin. Cheaper NOAC therapy with better safety and comparable efficacy to warfarin is the major requirement.
\end{abstract}

Keywords: NVAF, SPAF, Warfarin, NOAC

\section{INTRODUCTION}

Atrial Fibrillation (AF) is one of the commonest of cardiac arrhythmia. ${ }^{1}$ Large epidemiological studies have demonstrated that it is associated with different etiology, mainly valvular and non-valvular (NV). Among Nonvalvular etiologies, advancing age and cardiovascular conditions, particularly hypertensions and coronary artery disease, are common causes of non-valvular atrial fibrillation (NVAF). ${ }^{2}$

In the USA, approximately 2.3 million people have NVAF and this will be 3.3 million by 2020 and 5.6 million by $2050 .^{3}$ Non-valvular atrial fibrillations
(NVAF) will lead to increase in the risks of stroke and death. Oral anticoagulation therapy is very efficacious in prophylaxis of stroke and thereby reduces mortality in these patients. One of the oral anticoagulant, vitamin $\mathrm{K}$ antagonist warfarin, reduces the risks of stroke and death. Therefore, warfarin is recommended for patients who have atrial fibrillation and are at risk of having stroke. ${ }^{4}$

The 2010 European Society of Cardiologists (ESC) guidelines on risk of stroke associated with AF emphasized the use of the category, that is low, moderate and high-risk and recommended the use of anticoagulation therapy according to a risk factor-based approach, which can be expressed as an acronym, 
CHA2DS2-VASc [congestive heart failure/left ventricular dysfunction, hypertension, age $\geq 75$ (doubled), diabetes, stroke (doubled)-vascular disease, age (65-74), and sex category (female)]. Single score will be given to each present risk-factor in patients and double score to the age $\geq 75$ and previous attack of stroke. According to total final score, annual stroke risk will be classified in three categories, low risk (score $=0$ for male and 1 for female), moderate risk ( $(\mathrm{score}=1$ for male) and high risk (score $\geq 2)$. $^{5}$

The 'stroke prevention in atrial fibrillation' (SPAF) trial and a meta-analysis have shown that oral anticoagulation therapy (warfarin) is more effective than antiplatelet drug (low dose aspirin) in SPAF. Current guidelines suggests to give warfarin in AF patients having moderate and high risk for stroke (elderly, heart failure, etc.) according to CHA2DS2-VASc score, and to reserve aspirin for low risk patients or for those who are unable to take warfarin. Anticoagulation therapy is given for 3-4 weeks before and after attempting conversion of AF to sinus rhythm for SPAF. ${ }^{6}$

As warfarin is very effective in prophylaxis of SPAF, on the other side, it is associated with increase in the risk of hemorrhage. ${ }^{7}$ Warfarin is also cumbersome to use, because of their multiple interactions with food and drugs, and require frequent laboratory monitoring. These complications are real health care burden on patients, especially in developing country. Therefore, it is least used now a days, and when it is used, rates of discontinuation are high. ${ }^{8,9}$ Most of the patients receiving warfarin therapy also have inadequate anticoagulation. ${ }^{10}$ All these factors are serious disadvantage of using warfarin therapy.

For more than 50 years, vitamin $\mathrm{K}$ antagonists (eg, warfarin) were the only available oral anticoagulants. It has now been 5 years since the first of the new oral anticoagulants (NOACs), dabigatran etexilate, gained approval for stroke prevention in atrial fibrillation in the United States. This was followed by the approval of rivaroxaban and apixaban for this indication. ${ }^{11}$ Newer oral direct thrombin inhibitor like, dabigatran etexilate (dabigatran), was shown to be effective as an anticoagulant in the treatment of patients with atrial fibrillation in the randomized evaluation of long-term anticoagulation therapy (RE-LY) study. ${ }^{12,13}$ In another large trial dabigatran etexilate $150 \mathrm{mg}$ twice daily has yielded superior results to warfarin for prevention of embolism and stroke in patients of atrial fibrillation. ${ }^{6}$ These study reports of trials are signalling positive future of this drug in Indian market.

Serious complications associated with warfarin lead us to in urge of effective, safer and convenient newer anticoagulation agents over warfarin. So, the current questionnaire based study is done with the objectives to assess the prevalence of NVAF in Vadodara, Gujarat, to find out the unmet need and limitation of current oral anticoagulation therapy and also look out for the future of NOAC drugs for stroke prevention in atrial fibrillation (SPAF) patients.

\section{METHODS}

The proposed study was observational, non-interventional and prospective in nature. It was questionnaire based study, willingly participated by 20 cardiologists of Vadodara, India, to assess the prevalence of non-valvular atrial fibrillation (NVAF) in Vadodara, to find out unmet need and limitations of current anticoagulation therapy for SPAF and future of newer oral anticoagulation drugs (NOAC). The questionnaire used for the study purpose:

- What is the prevalence for NVAF (Non-Valvular Atrial Fibrillation) in Baroda?

- Would you prefer to give prophylaxis for stroke in all NVAF patients?

- How would you assess the need for stroke prophylaxis in NVAF patients?

- If yes, according to score, which drug therapy is given for prophylaxis for stroke in NVAF patients?

- What is your choice of anticoagulation therapy for stroke prophylaxis? And duration of therapy?

- What are the major side effects/problems you faced in the patients due to warfarin as anticoagulant therapy?

- Is warfarin safe to use or change is needed for stroke prophylaxis?

- How many numbers of times you have to give treatment/antidote due to warfarin induced bleeding?

- Are newer oral drug therapies preferable over older one, even if they have no specific antidote?

- What is ideal newer drug therapy for stroke prophylaxis? Better safety with comparable efficacy or better efficacy with comparable safety to warfarin.

\section{RESULTS}

According to questionnaire based study, we found that prevalence of NVAF (non-valvular atrial fibrillation) was around 2.5 to $3 \%$ in Vadodara, India.

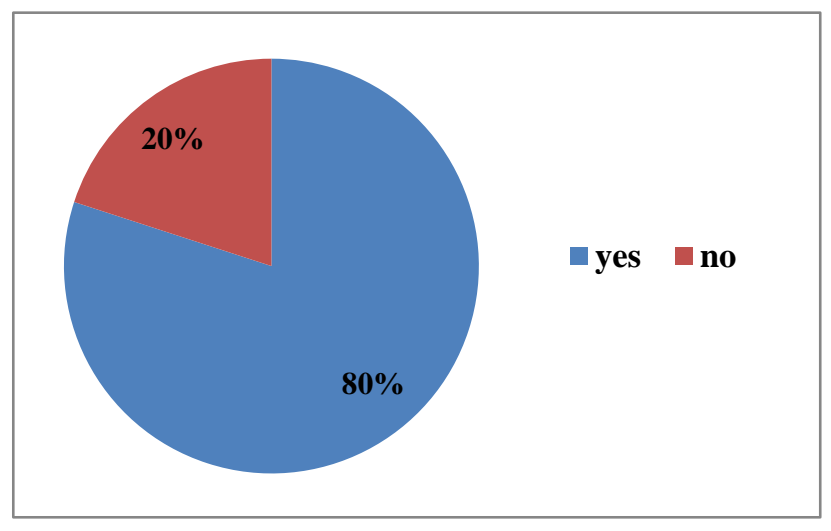

Figure 1: Prophylaxis given for SPAF by cardiologists. 
In study, total $80 \%(16 / 20)$ of cardiologists mentioned that prophylaxis is required in all the patients of NVAF for SPAF (stroke prevention in atrial fibrillation) (Figure 1).

All cardiologists (100\%), who prescribed prophylaxis for stroke, mentioned that prophylactic therapy for SPAF is given after evaluating CHA2DS2-VASc score and choice of therapy depends upon it (Table 1).

Table 1: Type of treatment according to CHA2DS2VASc score.

\begin{tabular}{|ll|}
\hline $\mathrm{CHA}_{2} \mathrm{DS}_{2}-\mathrm{VASc}$ score & $\begin{array}{l}\text { Type of } \\
\text { treatment }\end{array}$ \\
\hline $\begin{array}{l}0 \text { for male and } 1 \text { for female } \\
\text { (low risk) }\end{array}$ & $\begin{array}{l}\text { Antiplatelet drug } \\
\text { therapy }\end{array}$ \\
\hline$\geq 1$ for male and $\geq 2$ for female & Anticoagulation \\
(moderate and high risk) & drug therapy \\
\hline
\end{tabular}

In our study, all the cardiologists would like to prescribe antiplatelet drug (aspirin) as prophylaxis of choice for SPAF in "low risk score" (according to CHA2DS2-VASc score) patients, while "moderate and high risk scores" patients will be taken care by giving anticoagulation therapy like warfarin/NOACs.

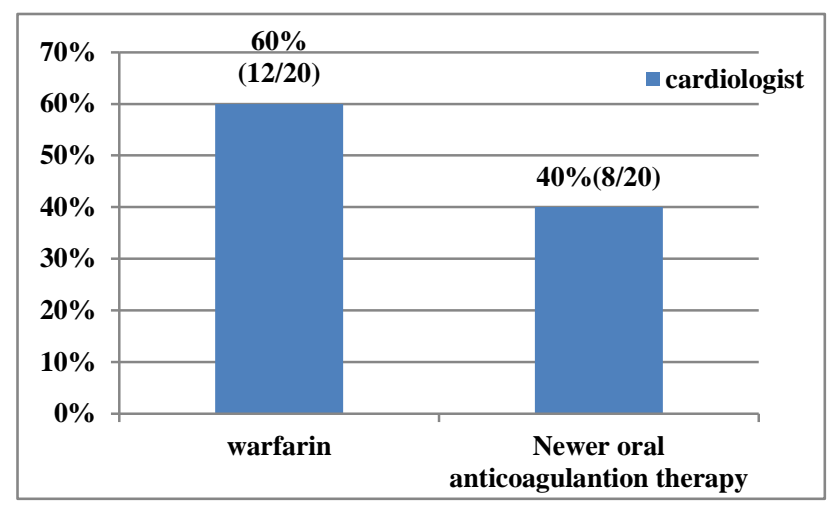

Figure 2: Anticoagulation therapy for SPAF by cardiologists.

Cardiologists were divided in their choice of anticoagulation therapy for SPAF. Total $60 \%$ of all cardiologists preferred to prescribe warfarin (Figure 2). Treatment for SPAF is given for lifelong by all cardiologists.

Majority of the cardiologists $(90 \%, 18 / 20)$ mentioned that both bleeding and continuous monitoring are the most commonly encountered problems associated with warfarin therapy. Other associated problems with warfarin therapy are, noncompliance to the patient, drug-drug interaction, longer duration and slow onset of action, from more severe to less severe one. According to cardiologists, approximately $40-50 \%$ of patients on warfarin therapy face any of the above mentioned problems during warfarin treatment.
In questionnaire based Study, all cardiologists thought that warfarin is unsafe anticoagulation therapy for SPAF due to its serious life threatening side effects and change is required. Cardiologists will prefer newer anticoagulation therapy (NOAC), if it provides fewer side effects over warfarin. Cardiologists, who prescribed NOAC, did not want to change their therapy.

Cardiologists, who are using warfarin for SPAF, had to give antidote (vitamin K) or FFP (fresh frozen plasma) to around $20 \%$ of total patients for controlling warfarin induced major or life threatening bleeding.

According to our study, all cardiologists thought that newer oral anticoagulation therapy without antidote can be accepted, if they are safe enough.

Total $90 \%$ of cardiologists thought that newer oral anticoagulant drugs must have better safety like less bleeding with comparable efficacy to warfarin. Remaining cardiologists thought that better efficacy with comparable bleeding to warfarin is desirable.

\section{DISCUSSION}

Atrial fibrillation, also known as "old" arrhythmia, has assumed increasing importance in the $21^{\text {st }}$ century. ${ }^{14}$ The growing "epidemic" of non-valvular atrial fibrillation (NVAF) with its associated morbidity and mortality is dangerous and intersects with a number of conditions including aging, thromboembolism, stroke, congestive heart failure, hypertension, and perhaps the metabolic syndrome and inflammation.

In the USA, approximately 2.3 million people have NVAF and this will be 3.3 million by 2020 and 5.6 million by $2050 .^{3}$ According to our survey, prevalence of NVAF was approximately 2.5 to $3 \%$ in Vadodara, Gujarata, India. Another study done by Ogawa S, Aonuma $\mathrm{K}$ et al found that prevalence of NVAF was $6.5 \%$ in India, while in Australia it was $16.9 \% .^{15}$

In our study, findings suggested that $80 \%$ of the total cardiologists would like to give prophylaxis to all the patients of NVAF for SPAF (stroke prevention in atrial fibrillation). Remaining $20 \%$ of the cardiologists would like to give prophylaxis in approximately $80 \%$ of total NVAF patients, which is also not less in number. Nonvalvular atrial fibrillation is a major cause of stroke and disability worldwide. ${ }^{16}$ The appropriate prophylactic medicines in patients with NVAF can reduce the rate of stroke by two-thirds or more. ${ }^{17}$ So it is beneficial to give prophylactic therapy to all NVAF patients for SPAF.

We observed in our study that cardiologists would like to give prophylaxis, to all the patients for SPAF, after assessing CHA2DS2-VASc score. The CHA2DS2-VASc score is inclusive of the most common risk factors for stroke, which are congestive heart failure/left ventricular dysfunction, hypertension, age $\geq 75$ (doubled), diabetes, 
stroke (doubled)-vascular disease, age (65-74), and Sex category (female). Single score is given to each present risk-factor in patients and double to age $\geq 75$ and previous attack of stroke. According to final total score, annual stroke risk will be classified in three categories, low risk (score $=0$ for male and 1 for female), moderate risk (score $=1$ for male) and high risk (score $\geq 2) .{ }^{18}$ The accumulated evidence shows that CHA2DS2-VASc is better at identifying 'truly low-risk' patients with AF and is as good as, and possibly better than, scores such as CHADS2 in identifying patients who develop stroke and thromboembolism. ${ }^{19,20}$ It is positive sign that cardiologists are assessing proper score to give prophylaxis for SPAF.

In our study, all the cardiologists would like to prescribe antiplatelet drug (aspirin) as prophylaxis of choice for SPAF in "low risk score" (according to CHA2DS2-VASc score) patients, while "moderate and high risk scores" patients will be taken care by giving anticoagulation therapy like warfarin. Since the publication of the 2010 ESC guidelines, additional evidence has also strengthened the use of prophylaxis therapy according to the risk factor-based approach (low, moderate and high) proposed in the guidelines, where 'truly low-risk' patients (aged $<65$ and lone AF) do not need any antithrombotic therapy, as they have very low absolute event rates, while both 'moderate and high risks' patients are taken care by giving oral anticoagulation therapy. ${ }^{21}$ So, findings in our study are similar to 2010 ESC guidelines regarding prophylaxis of choice for "moderate and high risks scores" patients.

We observed that cardiologists were divided in their choice of anticoagulation therapy for SPAF. Newer oral anticoagulation therapy is the treatment of choice for $40 \%$ of the total cardiologists. Remaining $60 \%$ of cardiologists, who prescribe warfarin, want to go for newer oral anticoagulation drugs, but high cost of it is the main obstacle. In India, currently vitamin $\mathrm{K}$ antagonist (VKA) drugs like warfarin remains the number one agent of choice for oral anticoagulation, due to years of usage and the prohibitive cost of newer oral anticoagulation drug. ${ }^{22}$ In the United States also, the much higher drug costs of newer oral anticoagulants (NOACs) taken into consideration by prescribers. High drug costs can contribute to reduced medication adherence of patients and lead to skip/split doses to make the medication last longer by them. ${ }^{23}$ Treatment for SPAF is also given lifelong. In such cases, higher cost of newer drug therapy is major burden on patients in developing country like India and makes compulsion for cardiologists to think twice before choosing newer drug therapy.

In our study majority of the cardiologists (90\%) stated both bleeding and continuous monitoring as main problems associated with warfarin therapy. According to other study done in India by Gopalakrishnan $\mathrm{S}$ et al mentioned that this certain issue, bleeding with warfarin, is more peculiar to Indians. Inconsistent consumption of green leafy vegetables like cabbage, cauliflower, spinach and other foods rich with vitamin $\mathrm{K}$ in the Indian diet would prevent the achievement of target INR on patients with warfarin and increase chances of bleeding. A lot of Indians are in the habit of taking over the counter medications [e.g. nonsteroidal anti-inflammatory drugs (NSAIDs)/tramadol] for various disorders from body aches to fever to jaundice. These medications would increase the anticoagulation action of the warfarin and may cause bleeding. Use of concomitant antituberculous drugs like isoniazid (INH) or rifampicin can also alter INR values and result in under or over anticoagulation. ${ }^{22}$ Continues difficulties in achieving target INR values, due to above mentioned reasons, will lead to continuous monitoring of therapy. Another Indian study regarding continuous monitoring of warfarin therapy, had shown that outpatient anticoagulant control was generally poor with an unacceptably high proportion of subtherapeutic PT/INR values and high complication rates. ${ }^{24}$ So bleeding and continuous monitoring are major drawbacks of warfarin therapy for SPAF.

There are also several other associated problems with therapy are, noncompliance to the patient, drug-drug interaction, longer duration and slow onset of action. According to study, approximately $40-50 \%$ of the patients face any of the above mentioned problems, which reduces the chances of continuing the same therapy and patients' adherence. Gopalakrishnan $\mathrm{S}$ et al. mentioned that around half of patients, who get warfarin, stop taking it especially in the developing world, and of those who still take it only half are in therapeutic range. So, only a small minority are well treated. $^{22}$

In a questionnaire based study, we got the feedback from cardiologists $(60 \%)$, who prescribed warfarin, that warfarin is unsafe anticoagulation therapy for SPAF due to its life threatening side effects and change is required accordingly. They will prefer newer oral anticoagulation (NOAC) therapy, only if it is cheaper and with lesser side effects over warfarin. Cardiologists $(40 \%)$, who prescribe newer oral anticoagulation therapy, did not want to change their choice of drug, according to study. Another study done by Ogawa s et al done in 6 different countries, mentioned that physicians prescribed dabigatran (NOAC) because of the higher efficacy and lower bleeding risk of dabigatran as compared with those of warfarin. This is evident from the data on the percentages assigned to the various reasons: efficacy, $77 \%$; bleeding risk, $67 \%$; and ease of administration, $55 \%$. The prescription of dabigatran may have been based on the results of the RELY trial. ${ }^{11,15}$ Some other study done by Moser M et al. mentioned that prohibitively high cost of newer oral anticoagulation (NOAC) drugs resulting in poor compliance of patients. ${ }^{25}$

We found that all cardiologists think that newer oral anticoagulation therapy without antidote can be accepted over warfarin, if they are safe enough. Bleeding associated with warfarin is mainly due to its mechanism of action (blocking vitamin $\mathrm{K}$ dependent clotting factors), 
longer half-life (36 to 42 hours) and longer duration of action ( 2 to 5 days) with improper monitoring. ${ }^{5}$ Therapy, for this bleeding, is either by omitting one or two doses of drug or by giving antidote (vitamin K). Vitamin $\mathrm{K}$ synthesizes the new vitamin K-dependent clotting factors, but it will take 6-24 hours. ${ }^{5}$ Mechanisms of action of newer oral anticoagulation drugs are different from warfarin and half-lives of drugs like dabigatran (12 to 14 hours), rivaroxaban (7 to 13 hours) and apixaban (8 to 13 hours) are shorter than warfarin. ${ }^{26}$ These factors may reduce the side effects like bleedings and reverse the drug action more rapidly, only by omitting the dose or stopping the treatment and counterbalance the need for antidote.

About $90 \%$ of total cardiologists think that newer oral anticoagulant drugs with better safety like less bleeding and comparable efficacy to warfarin. Only $10 \%$ of cardiologists think that better efficacy with comparable bleeding to warfarin is desirable. In the treatment of disease, both efficacy and safety are the important factors related to the drug. Treatment of disease is not justifiable if it will cause life threatening side effects. We have to change treatment plan from warfarin to other, if the side effects associated with drugs are serious. Warfarin is associated with major safety issues like life threatening bleeding. So, NOACs with better safety and comparable efficacy to warfarin is more desirable.

Overall, warfarin is an inexpensive generic medication, but regular INR monitoring and proper caring of patients due to related haemorrhagic complications is a substantial cost for healthcare systems. ${ }^{26}$ These are serious limitations of current anticoagulation therapy, warfarin. On the other side, higher cost of newer oral anticoagulation drug therapy (NOAC) makes it inappropriate to meet up the unmet need of current anticoagulation therapy in developing country like India.

\section{CONCLUSION}

Safety related issues, like life threatening bleeding, and Patients' noncompliance due to continuous INR monitoring are the main limitations of warfarin as anticoagulation therapy. Newer and cheaper oral anticoagulation drug therapy with better safety and comparable efficacy to warfarin is the major requirement of the society and will be welcomed by cardiologists as treatment of choice for stroke prophylaxis in NVAF patients.

Funding: No funding sources

Conflict of interest: None declared

Ethical approval: Not required

\section{REFERENCES}

1. Furberg CD, Psaty BM, Manolio TA. The prevalence of atrial fibrillation in elderly subjects. Am J Cardiol. 1994;74:236-41.
2. Jonas O, Jeff HS, Micheal E. Variations in cause and management of atrial fibrillation in a prospective registry of 15400 emergency department patients in 46 countries. The RE-LY Atrial Fibrillation Registry. Circulation. 2014;129:1568-76.

3. Bernard JG, Teresa SM, James BS. The changing epidemiology and natural history of non-valvular atrial fibrillation: clinical implications. Transactions of The American clinical and climatological association. 2004;115:149-60.

4. Fuster V, Ryden LE, Cannom DS. ACC/AHA/ESC 2006 guidelines for the management of patients with atrial fibrillation-executive summary: a report of the American college of cardiology/American Heart Association Task Force on Practice Guidelines and the European Society of Cardiology Committee for Practice Guidelines. J Am Coll Cardiol. 2006;48:854906.

5. Camm AJ, Kirchhof P, Lip GY, Schotten U, Savelieva I. Guidelines for the management of atrial fibrillation: the task force for the management of atrial fibrillation of the European Society of Cardiology (ESC). Europace. 2010;12:1360-420.

6. Tripathi KD. Drugs affecting coagulation, bleeding and thrombosis. Essentials of medical pharmacology. $7^{\text {th }}$ edition; 2013:44:613-633.

7. Hart RG, Pearce LA, Aguilar MI. Meta-analysis: antithrombotic therapy to prevent stroke in patients who have nonvalvular atrial fibrillation. Ann Intern Med. 2007;146:857-67.

8. Birman-Deych E, Radford MJ, Nilasena DS, Gage BF. Use and effectiveness of warfarin in Medicare beneficiaries with atrial fibrillation. Stroke. 2006;37:1070-4.

9. Hylek EM, Evans-Molina C, Shea C, Henault LE, Regan S. Major hemorrhage and tolerability of warfarin in the first year of therapy among elderly patients with atrial fibrillation. Circulation. 2007;115:2689-96.

10. Connolly SJ, Pogue J, Eikelboom J. Benefit of oral anticoagulant over antiplatelet therapy in atrial fibrillation depends on the quality of international normalized ratio control achieved by centers and countries as measured by time in therapeutic range. Circulation. 2008;118:2029-37.

11. Melnikova I. The anticoagulants market. Nat Rev. 2009;8(10):353-4.

12. Connolly SJ, Ezekowitz MD, Yusuf S, Reilly PA, Wallentin L. Newly identified events in the RE-LY trial. N Engl J Med. 2010;363:1875-6.

13. Eikelboom JW, Wallentin L, Connolly SJ. Risk of bleeding with 2 doses of dabigatran compared with warfarin in older and younger patients with atrial fibrillation: an analysis of the randomized evaluation of long-term anticoagulant therapy (RE-LY) trial. Circulation. 2011;123:2363-72.

14. Tsang TSM, Gersh BJ. Atrial fibrillation: an old disease, a new epidemic. American Journal of Medicine. 2002;113(5):432-5. 
15. Ogawa S, Aonuma K, Huang D, Huangd JL, Kalmane J, Kamakuraf S, et al. Fact-finding survey of antithrombotic treatment for prevention of cerebral and systemic thromboembolism in patients with nonvalvular atrial fibrillation in 9 countries of the AsiaPacific region. Journal of arrhythmia. 2012;28:41-55.

16. Wolf PA, Abbott RD, Kannel WB. Atrial fibrillation as an independent risk factor for stroke: the Framingham Study. Stroke. 1991;22:983-8.

17. Granger CB, Alexander JH, McMurray JJ, Lopes RD, Hylek EM, Hanna M, et al. ARISTOTLE committees and investigators. Apixaban versus warfarin in patients with atrial fibrillation. $\mathrm{N}$ Engl $\mathrm{J}$ Med. 2011;365:981-92.

18. Lip GY, Nieuwlaat R, Pisters R, Lane DA, Crijns HJ. Refining clinical risk stratification for predicting stroke and thromboembolism in atrial fibrillation using a novel risk factor-based approach: the Euro Heart Survey on Atrial Fibrillation. Chest. 2010;137:263-72.

19. Olesen JB, Torp-Pedersen C, Hansen ML, Lip G. The value of the CHA2DS2-VASc score for refining stroke risk stratification in patients with atrial fibrillation with a CHADS2 score 0-1: a nationwide cohort study. Thromb Haemost. 2012;107:1172-9.

20. Boriani G, Botto GL, Padeletti L, Santini M, Capucci A, Gulizia $\mathrm{M}$, et al. Improving stroke risk stratification using the CHADS2 and CHA2DS2VASc risk scores in patients with paroxysmal atrial fibrillation by continuous arrhythmia burden monitoring. Stroke. 2011;42:1768-70.

21. John AC, Gregory YH, Raffaele DC. Focused update of the ESC Guidelines for the management of atrial fibrillation. European Heart Journal. 2012;33:271947.

22. Gopalakrishnan S, Shrinivasan N. Oral anticoagulants: current Indian scenario. Medicine Update 2013;90:410-3. Available at http://www.apiindia.org/medicine_update_2013/chap 90.pdf. Accessed on 9 October 2015.

23. Choudhry NK, Saya UY, Shrank WH, Greenberg JO, Melia C, Bilodeau A, et al. Cost-related medication underuse: prevalence among hospitalized managed care patients. J Hosp Med. 2012;7(2):104-9.

24. Kakkar N, Kaur R, John M. Outpatient oral anticoagulant management: an audit of 82 patients. JAPI. 2005;53:847-52.

25. Moser $M$, Bode $C$. Anticoagulation in atrial fibrillation - a new era has begun. Hamostaseologie. 2012;32:37-9.

26. Kenneth AB. Pros and cons of new oral anticoagulants; Management of thromboembolic disease. Haematolgoy. 2013;2013:464-70.

Cite this article as: Mehta MD. Unmet need and limitations of current anticoagulation therapy for stroke prevention in atrial fibrillation patients: a questionnaire based study. Int J Basic Clin Pharmacol 2016;5:1471-6. 